128

\title{
LUNG DISEASE MORTALITY IN A COHORT OF STAFFORDSHIRE POTTERY WORKERS: FOLLOW-UP TO DECEMBER 2008
}

Nicola Cherry, ${ }^{1}$ Jessica Harris, ${ }^{2}$ Corbett McDonald, ${ }^{2}$ Susan Turner, ${ }^{3}$ Paul Cullinan, ${ }^{2}$ Tony Newman Taylor ${ }^{1}$ University of Alberta, Edmonton, Canada; ${ }^{2}$ Imperial College, London, UK; ${ }^{3}$ University of Manchester, Manchester, UK

\subsection{6/oemed-2011-100382.128}

Objectives To investigate deaths recorded as lung cancer or COPD (as distinct from other non-malignant respiratory disease), in a cohort occupationally exposed to silica.

Methods Vital status and cause of death were ascertained for a cohort of 5115 pottery workers previously followed to 1992. SMRs were calculated for underlying cause of death 1985-2008 compared to reference data from Stoke on Trent. Analysis of 'mentioned' cause of death was restricted to 19932008. Information on duration of employment and smoking was available for the whole cohort but exposure data were restricted to those included in an earlier validation cohort and those who were still employed in 1981 or later.

Results Of 5115 in the original cohort, 27 were duplicates, 29 were not traced and 258 had died before 1985, leaving 4801, with 1904 deaths. Deaths from lung cancer $(\mathrm{N}=243)$ were raised $S M R=1.15$ (1.01-1.30) as were deaths from COPD $(\mathrm{N}=158)$ both as an underlying (SMR=1.46 (1.24-1.71)) and as a 'mentioned' (SMR=1.31 (1.14-1.50)) cause. In a survival analysis duration of exposure was not related to deaths recorded as lung cancer or COPD for the cohort as a whole. In those $(\mathrm{N}=1943)$ with exposure data, early deaths (before July 1992) from either lung cancer or COPD were related to mean exposure in smokers but this relation was not seen in those dying from these causes in more recent years.

Conclusions Silica exposure appears to have contributed to early, but not recent, deaths although an excess of COPD deaths continues to be recorded. 